\title{
Do Risk Factors Explain the Increased Prevalence of Type 2 Diabetes Among California Asian Adults?
}

\author{
Sarah E. Choi • Vincent H. Chow $\cdot$ Sarah J. Chung • \\ Nathan D. Wong
}

Published online: 9 October 2010

(c) The Author(s) 2010. This article is published with open access at Springerlink.com

\begin{abstract}
Asian subgroup-specific information on type 2 diabetes mellitus (DM) is scarce. Using the California Health Interview Survey 2007 data, we examined Chinese, Korean, Japanese, Filipinos, and Vietnamese adults $(\mathrm{n}=$ $3,688)$ and Caucasian adults $(\mathrm{n}=33,981)$ for the prevalence of DM and risk factors. The age-adjusted prevalence of DM was the highest among Filipinos (8.05\%) followed by Japanese $(7.07 \%)$, Vietnamese $(7.03 \%)$, and Koreans (6.3\%). Chinese $(5.93 \%)$ was the only Asian group studied whose prevalence was lower than Caucasians (5.94\%). From multiple logistic regression, after adjusting for risk factors, Japanese had the highest likelihood of DM $(\mathrm{OR}=1.75, \mathrm{CI}=[1.12-2.73], P<0.05)$, followed by Filipinos (1.66, [1.13-2.43], $P<0.01$ ), and Koreans (1.59, [1.00-2.52], $P<0.05)$, relative to Caucasians. Our results suggest that even after accounting for lifestyle and other risk factor differences between Caucasians and key Asian subgroups in California, Japanese, Filipinos, and Koreans have a 1.6-1.75 greater likelihood of DM compared to Caucasians.
\end{abstract}

S. E. Choi $(\bowtie)$

Program in Nursing Science, College of Health Sciences, University of California, 214 Irvine Hall, Irvine, CA 92697-3959, USA

e-mail: sechoi@uci.edu

V. H. Chow · S. J. Chung · N. D. Wong

Heart Disease Prevention Program, Division of Cardiology, Department of Medicine, University of California, Irvine, CA, USA

e-mail: vchow@uci.edu

S. J. Chung

e-mail: chung.sarahj@gmail.com

N. D. Wong

e-mail: ndwong@uci.edu
Keywords Asians - Type 2 diabetes - Surveys · Epidemiology

\section{Introduction}

Diabetes mellitus is a metabolic disease characterized by hyperglycemia due to impaired insulin secretion, insulin metabolism, or both [1]. In adults, type 2 diabetes (DM), which is associated with impaired insulin metabolism, accounts for $90-95 \%$ of diabetes cases in the US and most industrialized countries [2,3]. Asian Americans are susceptible to developing diabetes, and the incidence is rapidly rising in this population $[4,5]$. The National Health Interview Survey 2007 indicates the prevalence of diabetes in Asians as $8.9 \%$ which is 1.4 times higher than Caucasians [6]. According to a recent study conducted in California where the largest population of Asians in the country reside [7], Asians experienced the second largest increase in diabetes prevalence, next only to American Indians between 2001 and 2005 [8].

Risk factors for DM identified by the American Diabetes Association include age, body mass index (BMI), exercise habits, history of hypertension, and dyslipidemia [9]. In addition, socioeconomic and cultural factors such as health insurance, poverty level, and acculturation have been linked to the prevalence of diabetes among minority populations [10-12]. Despite the increasing DM prevalence and rates of population growth of Asian Americans [7], little is known about ethnic-specific DM risks of this group; it is unclear whether risk factors identified among Caucasians are equally applicable to Asian Americans. Furthermore, Asian Americans are a heterogeneous group with diverse culture, health practices, and lifestyles [7], yet there is inadequate information regarding DM risk factors within each Asian subgroup. 
Aggregate health data may mask important heterogeneity in risk factor profiles among different Asian subgroups and thus prevent from identifying vulnerable populations for intervention. Therefore, we examined the prevalence of DM within a sample of California adults from five Asian ethnic groups and Caucasians, and examined associated risk factors in those with DM. Our specific aims were: (1) to describe the prevalence of DM among California Asians and Caucasians; (2) to describe prevalence of lifestyle and clinical risk factors among different Asian groups and Caucasians with DM; and (3) to examine whether risk factor differences explain the excess prevalence of DM in certain Asian subgroups compared to Caucasians.

\section{Methods}

Using the California Health Interview Survey (CHIS) 2007 [13], we examined California adults aged 18 and older ( $\mathrm{n}=50,555$ projected to 26.6 million) to determine DM likelihood among different Asian ethnicities relative to Caucasians. The CHIS is a biennial population-based telephone interview health survey of individuals residing in households in California. The survey collects a variety of health information including diseases, lifestyle and health behaviors, health status, socioeconomic status, and access to healthcare. The CHIS sample was drawn from all of California's 58 counties and was designed to be representative of the diversity of the California population. The sample is weighted to represent the non-institutionalized population for each sampling stratum and statewide. The weighting procedure used for CHIS 2007 compensates for differential probabilities of selection for households and persons, reduces biases occurring because non-respondents may have different characteristics than respondents, adjusts for under-coverage in the sampling frames, and reduces the variance of the estimates by using auxiliary information.

One randomly selected adult was interviewed per household. Interviews were conducted in multiple languages (English, Spanish, Mandarin, Cantonese, Vietnamese, and Korean). Questions related to health conditions, health behaviors (smoking, daily fruit and vegetable consumption, and vigorous exercise), poverty level, and health insurance coverage were asked. BMI was calculated in $\mathrm{kg} / \mathrm{m}^{2}$ based on self-reported height and weight without shoes. Ethnicity was defined by participants' self-report. Type 2 diabetes (DM) was defined by the respondent's selfreported answer choice of type 2 diabetes to the question "Were you told that you had type 1 or type 2 diabetes?" The respondent was aided by the interviewer's description of type 1 and type 2 diabetes, if needed. Finally, hypertension, heart disease, and heart failure were also defined by selfreport or having been told by doctor to have the condition.
The Chi-square test of proportions and analysis of variance (ANOVA) were used to compare the prevalence of DM and risk factors across the different ethnicities. Multivariable logistic regressions were used to determine which risk factors and Asian subgroups (relative to Caucasians) remained independently associated with an increased likelihood of having DM. Similar analyses were also conducted to examine the risk factors associated with the likelihood of DM within each Asian subgroup. SAS version 9.1.3 (SAS institute, Cary, NC) and SUDAAN software (RTI International, Research Triangle Park, North Carolina) were used for analysis and computation of weighted estimates for projection to the California population.

\section{Results}

An analysis of the prevalence of DM in Californian adults (Fig. 1) demonstrates that the age-adjusted prevalence of DM was the highest among Filipinos $(8.05 \%)$ followed by Japanese (7.07\%), Vietnamese (7.03\%), and Koreans (6.3\%). Chinese (5.93\%) was the only Asian group studied whose prevalence was comparable to Caucasians $(5.94 \%)$. The difference in DM prevalence among the groups was significant $(P<0.05)$.

In our sample of Californian Asians and Caucasian adults with DM, gender, being US-born, duration of living in US, insurance, poverty level, smoking, overweight, obesity, congestive heart failure, and heart disease prevalence were significantly different across ethnicity groups $(P<0.01)$. Vigorous activity within the last 7 days was also significantly different across the different ethnic groups $(P<0.05)$ (Table 1$)$.

Multiple logistic regression examining the likelihood of DM with covariates including various disease, demographic, and lifestyle factors (Table 2), showed that Japanese, Filipinos, and Koreans were at greater odds of having

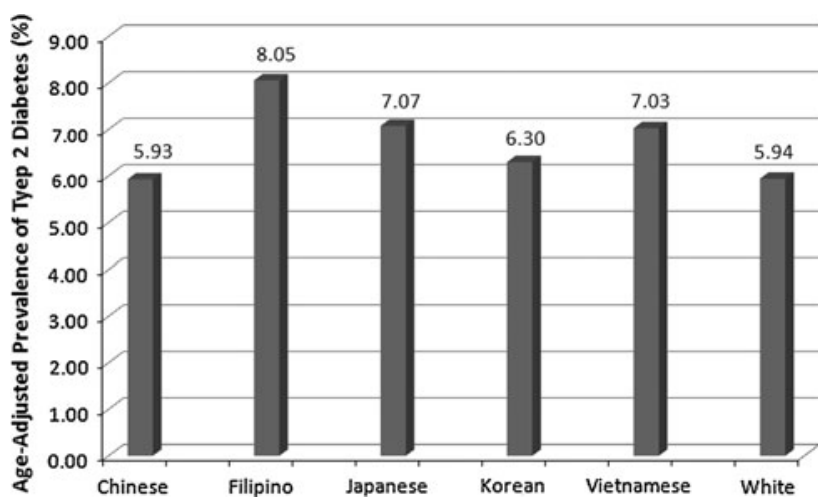

Fig. 1 Age-adjusted prevalence (in percent) of DM among Adults $\geq 18$ years old in California. California Health Interview Survey 2007 by Ethnicity. $P<0.05$ across ethnic groups 
DM than Caucasians. Japanese had the greatest odds of DM (odds ratio $(\mathrm{OR})=1.75$, confidence interval $(\mathrm{CI})=$ [1.12-2.73], $P<0.05)$. Filipinos and Koreans had also more than a 1.5-fold greater likelihood than Caucasians of DM (1.66, [1.13-2.43], $P<0.01$ and 1.59, [1.00-2.52], $P<0.05$, respectively). Furthermore, increased age, being male, being insured, having hypertension, more times eating vegetables per week, lack of vigorous exercise in the past week, and increased body mass index (BMI) were all associated with an increased likelihood of DM. A stepwise logistic regression further examined the factors contributing to the greater likelihood for DM in Japanese, Filipinos, and Koreans compared to Caucasians. Beginning with ethnicity, we subsequently adjusted for each of the factors included in the logistic regression models. When BMI was added to the model, Koreans, Japanese, and Filipinos emerged as having an increased odds of DM compared to Caucasians that was not apparent before adjustment, indicating that these important differences in DM are revealed once ethnic differences in BMI are accounted for.

\section{Discussion}

We found that among five Asian subgroups and Caucasians in California the age-adjusted prevalence of DM was the highest in Filipinos $(8.05 \%)$ followed by Japanese (7.07\%), Vietnamese (7.03\%), and Koreans (6.3\%). Chinese $(5.93 \%)$ was the only Asian group studied whose prevalence was similar to Caucasians (5.94\%). We also showed that there were significant differences in the prevalence of certain risk factors, lifestyle behaviors, and co-morbidities among Asian subgroups and Caucasians with DM.

Since most national diabetes statistics and epidemiologic studies for adults do not differentiate type 1 and type 2 diabetes, it is difficult to compare our prevalence for type 2 diabetes with others. However, compared with a recent national 7.5\% prevalence (not specific for type 2) reported for Asian Americans as an aggregate [14], the prevalence of DM observed in this study for Filiponos was higher. The higher prevalence of DM among Filipinos compared to Caucasians may be related to socioeconomic status, lifestyle behaviors, and co-morbidities that place individuals in this group at higher risk for DM. For example, Filipinos with DM had a greater percentage of being below the federal poverty level (FPL), which suggests the possibility of unhealthy eating (e.g., fast foods consumption), poor nutrition, lack of exercise (e.g., due to unsafe neighborhoods), access to preventive care, all associated with obesity and DM. Higher prevalence of overweight, CHF, and heart disease in this group supports the higher prevalence of DM among this group.

We found that Japanese were 1.75 times more likely to have DM compared to Caucasians, followed by Filipinos and Koreans both of which had over a 1.5 greater likelihood than Caucasians. These results are consistent with epidemiological studies reporting Japanese-American elderly in Hawaii to have double the prevalence of DM than nonHispanic whites [15], and the estimated prevalence of DM among Filipinos, Chinese, Japanese, and Koreans in Hawaii to be at least twice that of Caucasians [5]. In addition to ethnicity, increased age, being male, being insured, having high blood pressure, more vegetable consumption per week, lack of vigorous exercise in the past week, and higher BMI were all associated with an increased likelihood of DM. These risk factors are consistent with those identified by previous studies with Caucasian, Mexican Americans, and Japanese Americans [16-19].

We attempted to explain the excess odds of DM among Japanese, Filipinos, and Koreans by risk factor differences. The addition for BMI in the stepwise regression revealed that Koreans, Japanese, and Filipinos had a 1.6-1.75 greater odds of DM than Caucasians that was not apparent before adjustment. This suggests that the generally lower BMI in these ethnic groups compared to Caucasians was obscuring differences in DM likelihood between these Asian groups and Caucasians; these differences are revealed once BMI is put on "equal basis" between all ethnic groups. In other words, at a same level of BMI, Asians may be more susceptible to diabetes than Caucasians.

We could not definitively determine why Japanese are at a higher likelihood for DM than other Asian subgroups and Caucasians. BMI differences may in part explain this, although the prevalence of overweight and obesity among Japanese in our study were lower than other subgroups except Vietnamese. This finding suggests that among Japanese other risk factors than traditional obesity measured by BMI (e.g., abdominal adiposity, genetic factors) may play a role in their increased odds of DM.

While excess odds for DM in Filipinos may be explained by this group's high prevalence of overweight and obesity as well as co-morbidities, among Koreans, socioeconomic and cultural factors may contribute to their excess odds for DM. Consistent with a previous report [8], Koreans with DM in this study had the lowest prevalence of being born in the US and being insured. Lack of health insurance and limited English language proficiency are related to the prevention and outcome of DM in minority immigrant populations [20-22]. The high prevalence and odds for DM among Koreans in California may be related to limited access to preventive health care and lack of Korean language health education materials.

Koreans with DM showed the second highest overweight rate next to Filipinos, and as in Filipinos and Japanese, the prevalence of overweight was much higher than obesity. This is consistent with previous studies suggesting that Asians develop DM at a lower BMI than Caucasians due to a 


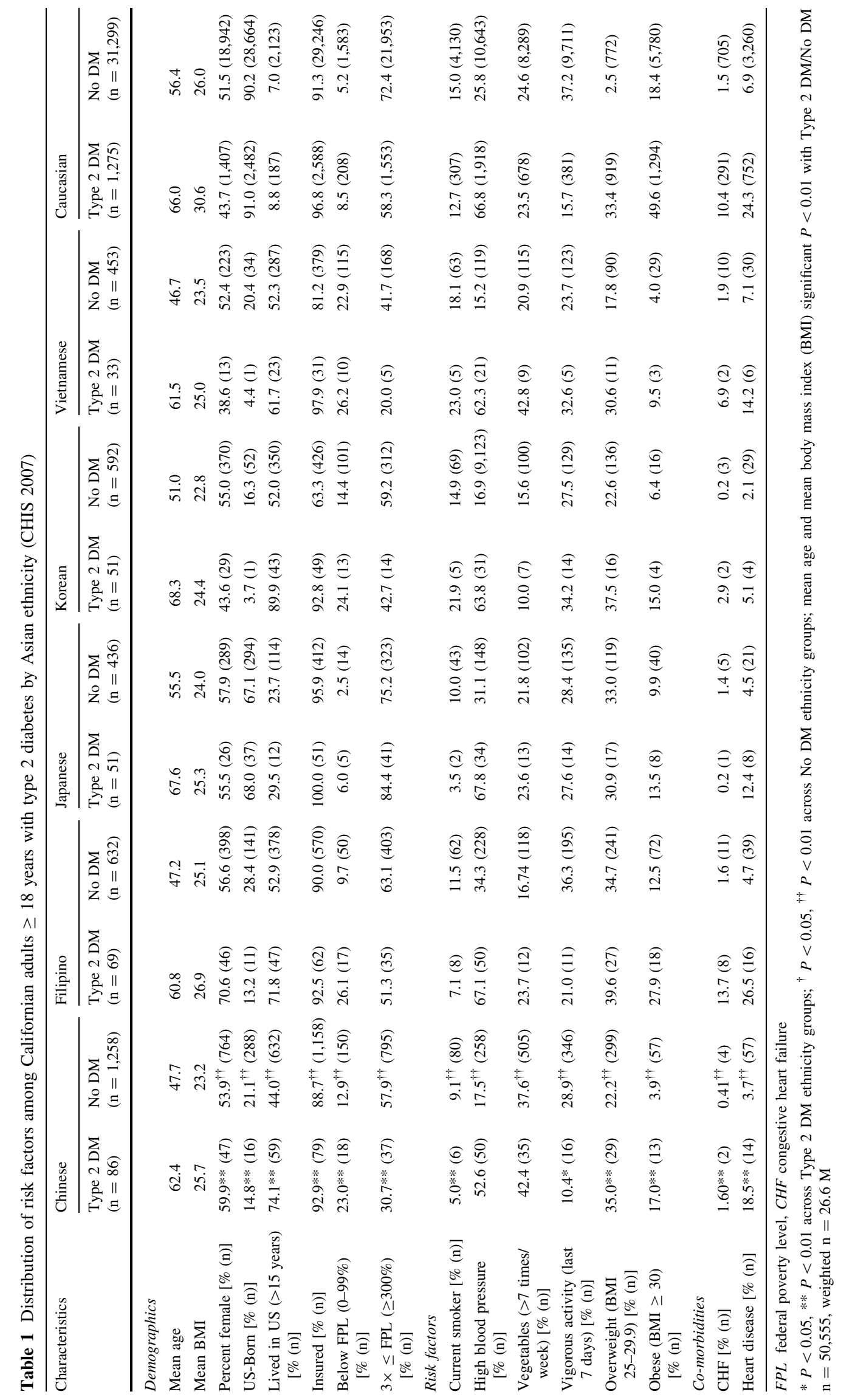


Table 2 Logistic regression examining ethnicity and other factors in relation to the likelihood of type 2 diabetes (CHIS 2007)

\begin{tabular}{lll}
\hline Variables & $\begin{array}{l}\text { Odds } \\
\text { ratio }\end{array}$ & $\begin{array}{l}\text { Confidence } \\
\text { interval }\end{array}$ \\
\hline Chinese vs. Caucasian & 1.38 & $0.97-1.96$ \\
Filipino vs. Caucasian & $1.66^{* *}$ & $1.13-2.43$ \\
Japanese vs. Caucasian & $1.75^{*}$ & $1.12-2.73$ \\
Korean vs. Caucasian & $1.59^{*}$ & $1.00-2.52$ \\
Vietnamese vs. Caucasian & 1.52 & $0.87-2.67$ \\
Age (in 10 year increments) & $1.58^{* *}$ & $1.51-1.64$ \\
Male vs. Female & $1.49^{* *}$ & $1.30-1.71$ \\
Insured vs. Uninsured & $1.65^{* *}$ & $0.44-0.83$ \\
Below federal poverty level & 1.24 & $0.94-1.64$ \\
$\quad$ (0-99\% vs. 100-199\%) & & \\
Current smoker vs. non-smoker & 1.18 & $0.97-1.43$ \\
High blood pressure (yes vs. no) & $2.32^{* *}$ & $1.98-2.71$ \\
Times eating vegetables (per week) & $1.01^{*}$ & $1.00-1.03$ \\
Vigorous physical activity & $0.79^{* *}$ & $0.67-0.94$ \\
$\quad$ (last 7 days yes vs. no) & & \\
BMI & $1.12^{* *}$ & $1.11-1.14$ \\
\hline
\end{tabular}

$* P<0.05 ; * * P<0.01$

difference in proportion of body fat to BMI [23, 24]. A study using Asian BMI criteria [25] also reported that $38 \%$ of the general Korean population in California (including those with DM) were overweight (BMI 23-27.4 $\mathrm{kg} / \mathrm{m}^{2}$ ) and only $7.5 \%$ were obese $\left(\mathrm{BMI} \geq 27.5 \mathrm{~kg} / \mathrm{m}^{2}\right)$. The association of lower BMI and higher DM odds was also observed in a recent population-based health survey, in which similar proportions of Asian and non-Hispanic Whites were reported to have DM initially, yet after accounting for the BMI limits for Asians, the adjusted prevalence of DM was $60 \%$ higher in Asian Americans [26].

The high prevalence of DM among U.S. Asians has been related to the adoption of a Western lifestyle, including a diet high in fat, processed foods, and less physical activity, which led to dramatic rise of obesity [15, 27, 28]. However, our study showed some inconsistencies; although Japanese were most likely to be engaged in a Western lifestyle given that $2 / 3$ of the study sample were U.S. born, they were not the most obese group, yet had the highest odds for DM. Similarly, Chinese exercised the least, yet this group had the lowest DM likelihood compared to Caucasians. Our findings suggest that traditional risk factors defined for Caucasians may not fully explain DM risk among Asian Americans. Other socio-economic, cultural, and environmental factors may play a role in DM likelihood among Asian subgroups. Further study is needed to examine the impact of socio-economic and cultural factors on Asian immigrants' risk for DM.

Our findings also suggest that prevalence rates may not necessarily reflect the risk factor-adjusted likelihood of
DM. While the prevalence of DM among Japanese in California is lower than that of Filipinos, Japanese had the greatest risk factor-adjusted odds of DM among Asians when compared with Caucasians. This indicates the need to look beyond lifestyle and traditional risk factors, such as investigating inflammatory, genetic, or other novel risk factors that might help explain these differences. In addition, increased attention for preventive measures is needed for Japanese to reduce the further rise of DM in this group.

Limitations of our secondary analysis study include using an existing dataset based on self-report rather than objective measures. Self-report may be subject to recall bias, which may have impacted the accuracy of the information on DM prevalence by false-negative error and the underestimation of DM prevalence rates. The survey design excluded individuals using cell phones, or those hospitalized and institutionalized from participating. Asians in the CHIS survey were categorized into eight groups (Chinese, Filipino, Korean, Vietnamese, Japanese, South Asian, Other Asian, and More than one Asian ethnic group). We focused our study on homogeneous Eastern and Southeastern Asians and therefore heterogeneous populations (e.g., South Asian) were excluded. Strengths of our study include Asian subgroup specific health data obtained in individuals' native languages, and our ability to project our findings to Asian subgroup populations in California.

In conclusion, the age-adjusted prevalence of DM and risk factors vary among Asian subgroups in California. Compared with Caucasians, Japanese had the greatest risk factor-adjusted odds of DM, followed by Filipinos and Koreans. The excess odds of DM in these subgroups were not explained by traditional risk factors. The findings highlight the need to study Asian subgroups individually, not as an aggregate, and to consider socio-economic, cultural, environmental factors as well as genetics and other novel risk factors when assessing DM risk and prevalence among Asian subgroups.

Open Access This article is distributed under the terms of the Creative Commons Attribution Noncommercial License which permits any noncommercial use, distribution, and reproduction in any medium, provided the original author(s) and source are credited.

\section{References}

1. Expert Committee on the Diagnosis and Classification of Diabetes Mellitus. Report of the expert committee on the diagnosis and classification of diabetes mellitus. Diabetes Care. 2003;26 (Suppl 1):S5-20.

2. Mann JI. Diet and risk of coronary heart disease and type 2 diabetes. Lancet. 2002;360(9335):783-9.

3. National diabetes statistics, 2007. National institutes of health publication No. 08-3892 June 2008. Available at www.diabetes. niddk.nih.gov. 
4. The diabetes epidemic among Asian Americans and Pacific Islanders. National diabetes education program. Available at http://ndep.nih.gov/media/Diabetes_AsianAm.pdf. Accessed 13 Aug 2009.

5. Caballero AE. Diabetes in minority populations. In: Kahn CR, Weir GC, King GL, Jacobson AM, Moses AC, Smith RJ, editors. Joslin's Diabetes Mellitus. 14th ed. Philadelphia: Lippincott, Williams \& Wilkins; 2005. p. 505-24.

6. Pleis JR, Lucas JW. Summary health statistics for U.S. adults: national health interview survey, 2007. Vital Health Stat 10. 2009;10(240):1-159.

7. U. S. Census Bureau. The Asian and Pacific Islander population in the United States: March 2000 (Update) (PPL-146). Available at http://www.census.gov/population/www/socdemo/race/ppl-146. html. Accessed 7 Mar 2009.

8. Diamant AL, Babey SH, Hastert TA, Brown ER. Diabetes: the growing epidemic. Policy Brief UCLA Cent Health Policy Res. 2007;(PB2007-9):1-12.

9. American Diabetes Association. Standards of medical care in diabetes-2009. Diabetes Care. 2009;32(Suppl 1):S13-61.

10. Perez-Escamilla R, Putnik P. The role of acculturation in nutrition, lifestyle, and incidence of type 2 diabetes among Latinos. J Nutr. 2007;137(4):860-70.

11. Centers for disease control and prevention. Diabetes among hispanics-Los Angeles County, California, 2002-2003. MMWR Morb Mortal Wkly Rep. 2003;52 (47):1152-5.

12. Signorello LB, Schlundt DG, Cohen SS, Steinwandel MD, Buchowski MS, McLaughlin JK, et al. Comparing diabetes prevalence between African Americans and Whites of similar socioeconomic status. Am J Public Health. 2007;97(12):2260-7.

13. California Health Interview Survey. CHIS 2005 adult public use file, release 1 [computer file]. Los Angeles, CA: UCLA center for health policy research;2007.

14. National Institute of Diabetes and Digestive and Kidney Diseases. National diabetes statistics, 2007 fact sheet. Available at http://diabetes.niddk.nih.gov/DM/PUBS/statistics/. Accessed 19 Apr 2009.

15. Rodriguez BL, Curb JD, Burchfiel CM, Huang B, Sharp DS, Lu GY, et al. Impaired glucose tolerance diabetes and cardiovascular disease risk factor profiles in the elderly. The honolulu heart program. Diabetes Care. 1996;19(6):587-90.

16. Stern MP, Williams K, Haffner SM. Identification of persons at high risk for type 2 diabetes mellitus: do we need the oral glucose tolerance test? Ann Intern Med. 2002;136(8):575-81.
17. Fujimoto WY, Bergstrom RW, Boyko EJ, Chen K, Kahn SE, Leonetti DL, et al. Type 2 diabetes and the metabolic syndrome in Japanese Americans. Diabetes Res Clin Pract. 2000;50(Suppl 2):S73-6.

18. Goel MS, McCarthy EP, Phillips RS, Wee CC. Obesity among US immigrant subgroups by duration of residence. JAMA. 2004;292(23):2860-7.

19. Gordon-Larson P, Harris KM, Ward DS, Popkin BM. Acculturation and overweight-related behaviors among Hispanic immigrants to the US: the national longitudinal study of adolescent health. Soc Sci Med. 2003;57(11):2023-34.

20. Solis J, Marks G, Garcia M, Shelton D. Acculturation, access to care, and use of preventive services by Hispanics: findings from HHANES 1982-84. Am J Public Health. 1990;80(Suppl):11-9.

21. Perez-Stable EJ, Napoles-Springer A, Miramontes JM. The effects of ethnicity and language on medical outcomes of patients with hypertension or diabetes. Med Care. 1997;35(12):1212-9.

22. Hsu WC, Cheung S, Ong E, Wong K, Lin S, Leon K, et al. Identification of linguistic barriers to diabetes knowledge and glycemic control in Chinese Americans with diabetes. Diabetes Care. 2006;29(2):415-6.

23. Deurenberg P, Deurenberg-Yap M, Guricci S. Asians are different from Caucasians and from each other in their body mass index/body fat per cent relationship. Obes Rev. 2002;3(3):141-6.

24. Steering committee of the western pacific region of the world health organization, the international association for the study of obesity, and the international obesity task force. The Asia-Pacific perspective: redefining obesity and its treatment. Melbourne, Australia: health communications Australia, 2000.

25. Cho J, Juon HS. Assessing overweight and obesity risk among Korean Americans in California using world health organization body mass index criteria for Asians. Prev Chronic Dis. 2006; 3(3):1-11.

26. McNeely MJ, Boyko EJ. Type 2 diabetes prevalence in Asian Americans: results of a national health survey. Diabetes Care. 2004;27(1):66-9.

27. Frisbie WP, Cho Y, Hummer RA. Immigration and the health of Asian and Pacific Islander adults in the United States. Am J Epidemiol. 2001;153(4):372-80.

28. Song YJ, Hofstetter CR, Hovell MF, Paik HY, Park HR, Lee J, et al. Acculturation and health risk behaviors among Californians of Korean decent. Prev Med. 2004;39(1):147-56. 